Voix et Images

volxetimages

\title{
Littérature populaire religieuse : Esquisse sociopsychanalytique d'un héros : Gérard Raymond
}

\section{Claude-Marie Gagnon}

Volume 6, numéro 3, printemps 1981

Philippe Haeck

URI : https://id.erudit.org/iderudit/200286ar

DOI : https://doi.org/10.7202/200286ar

Aller au sommaire du numéro

Éditeur(s)

Les Presses de l'Université du Québec

ISSN

0318-9201 (imprimé)

1705-933X (numérique)

Découvrir la revue

Citer cet article

Gagnon, C.-M. (1981). Littérature populaire religieuse : Esquisse

sociopsychanalytique d'un héros : Gérard Raymond. Voix et Images, 6(3),

465-472. https://doi.org/10.7202/200286ar d'utilisation que vous pouvez consulter en ligne.

https://apropos.erudit.org/fr/usagers/politique-dutilisation/ 


\title{
Littérature populaire religieuse: Esquisse sociopsychanalytique d'un héros: Gérard Raymond
}

\author{
par Claude-Marie Gagnon
}

\begin{abstract}
J'ai commencé à savourer les délices spirituels de Une âme d"élite: Gérard Raymond. Quelle éloquente prédication pour notre jeunesse étudiante et ouvrière! Car celle-ci pourra puiser de grandes leçons à l'égard de la jeunesse étudiante dans la vie de ce jeune homme sorti d'un milieu ouvrier.

Un supérieur de patronage. Cité dans: Gérard Raymond, Journal, Québec, I'Action Catholique, 1938, p. 181.
\end{abstract}

Depuis quelques années, on assiste à un essor des recherches en paralittérature québécoise. En effet, quelques courageux pionniers ont entrepris de mettre en valeur les coutumes épistolaires ${ }^{1}$ et familiales d'antan ${ }^{2}$, I'histoire de notre production radiophonique ${ }^{3}$, télévisuelle ${ }^{4}$ et theâtrale ${ }^{5}$. Même le contenu de nos journaux à potins a été décortiqué avec humour et sérieux ${ }^{6}$. Quant au plus célèbre de nos héros populaires, l'agent secret Ixe-13, n'a-t-il pas été rappelé à la mémoire des Québécois par le film de Jacques Godbout ${ }^{7}$ et par une vaste recherche effectuée par le département des littératures de I'Université Laval ${ }^{8}$.

Mais il y a tout un domaine de notre patrimoine culturel que l'avènement de la révolution tranquille a délibérément rejeté dans l'ombre comme un mauvais souvenir et qui a été encore peu exploré. Jean Simard en parle comme d'un "patrimoine méprisé ${ }^{9}$ tandis que Victor-Lévy Beaulieu y fait allusion en le qualifiant de "littérature underground ${ }^{10}$ ». II s'agit d'un ensemble romanesque d'inspiration catholique qu'on pourrait désigner du nom de "paralittérature religieuse»"1. Pratiquement, cette dernière regroupe tous les ouvrages qui font partie de ce qui semble constituer la abibliothèque bleue ${ }^{12}$ des Québécois, en particulier les nombreuses biographies et autobiographies de candidats québécois à la sainteté. 
Car la plupart des Québécois adultes se souviennent d'une enfance où on leur recommandait fortement la lecture d'un certain nombre de vies romancées de petits Québécois modèles. On ne peut douter que ces vies de jeunes saints, qui ont connu des tirages plus importants que certains classiques de la littérature québécoise ${ }^{13}$, de même que le célèbre Petit Catéchisme gris en usage dans tous les diocèses de la province, aient contribué à former la littérature québécoise telle que nous la connaissons aujourd'hui. Ne pourrait-on pas soupçonner que des succès comme le Journal de Gérard Raymond ${ }^{14}$ ou Hors de sa prison ${ }^{15}$, par exemple, ont constitué le terreau d'où ont germé des œuvres comme Les enfants du sabbat d'Anne Hébert ou Une saison dans la vie d'Emmanuel de Marie-Claire Blais? Et la grande diffusion de cette paralittérature religieuse n'aurait-elle pas contribué, à sa façon, au fort courant anticlérical qu'on relève dans la littérature québécoise des années 1940$1960^{16}$ ?

Une étude idéologique de cette paralittérature religieuse n'aurait probablement aucune peine à y retracer des marques de l'idéologie de conservation dont elles sont de purs produits et d'ardentes propagandistes. Par contre, l'examen de la structure fantasmatique se révélerait peut-être autrement intéressant ${ }^{17}$. Pourtant ces œuvres, qui érigent la soumission et la souffrance en modèles de vie, ont-elles été consommées par une fraction importante de la société québécoise ${ }^{18}$ ? L'influence du clergé est évidente mais n'explique pas tout. Le clergé pouvait bien contrôler les média d'information, les livres, les manuels scolaires - où le groupe québécois était défini comme un peuple qui a une histoire édifiante qu'il importe de conserver intacte - il ne pouvait quand même pas contrôler l'inconscient de ses ouailles, ni celui de ses membres.

Il pouvait bien sûr, par le biais de ses appareils idéologiques, inculquer la terreur du péché et la maladie du scrupule, manifestations du refoulement de pulsions jugées coupables. Mais si ces mêmes pulsions coupables avaient ressurgi, à peine travesties, dans ces œuvrettes pieuses dont le clergé vantait aveuglément la lecturé? Pourrait-on soupçonner la coïncidence entre le fantasme d'un auteur - Gérard Raymond dans le cas qui nous intéresse et le désir, maladroitement refoulé à coup de chemins de croix et de apremiers vendredis du mois", d'une partie de la société québécoise ${ }^{19}$ ? Est-ce que, malgré leur conservatisme bien apparent, ces romans religieux auraient été le principal exutoire à un désir dont l'analyse du Journal de Gérard Raymond permettrait de préciser la nature?

Manifestement, ces «vies de saints», dont le Journal est un représentant type, présentent une structure identique. Elles mettent en scène un héros qui se destine à la vie religieuse et aspire de toutes ses forces à une sainteté crucifiante. Ledit héros est bientôt frappé par la tuberculose - ou une maladie analogue - et meurt dans la fleur de l'âge, embrasé par l'amour divin. Les proches du héros défunt - généralement des religieux - entreprennent de rédiger sa biographie et de la diffuser, espérant gagner de nouveaux candidats à la sainteté. Mais dans certains cas, le héros, pressentant sa mort prochaine 
et possédant certains dons littéraires, a rédigé son autobiographie. C'est le cas de Gérard Raymond.

Gérard, le narrateur ${ }^{20}$, entreprend d'écrire son journal à la suite de la lecture de la biographie de Paul-Émile Lavallée ${ }^{21}$. En apparence, le journal glisse rapidement sur la situation familiale. Pourtant, une page entière est consacrée par Gérard à la description des sentiments qu'il éprouve envers sa mère: «c'est à elle, après Dieu, que je dois tout ce que je possède de piété, de vertus. C'est grâce à elle que je me suis bien conservé. C'est grâce à ses prières, à ses sacrifices que le péché s'est écarté de moi et que Dieu m'a rempli de ses grâces" (p. 7). Par contre, le père ne jouit pas d'un crédit aussi favorable et on sent percer chez Gérard une pointe d'agressivité à l'égard de ce dernier: «ll n'a pas tout à fait la même méthode que ma mère, il n'a pas surtout la grande bonté de celle-ci» (id.). Mais cette agressivité est refoulée car une seule attitude est tolérée envers ce père rude, comme pour neutraliser une éventuelle réplique: «je veux lui faire plaisir le plus possible, lui être bien soumis, bien obéissant» (p. 8).

II adopte la même attitude envers ces représentants de l'image paternelle que sont les professeurs du Séminaire et monsieur le Curé, qui recommandent tous une même chose à ce futur missionnaire: le travail, «même pendant les vacances du Jour de l'An» (p. 9). II faut dire que le héros, élève modèle, ne cache nullement ses ambitions: "Je suis au Séminaire en Troisième. C'est déjà pas mal pour quinze ans. De plus, je me place très bien en classe [car] je veux profiter du talent que Dieu m'a donné, le faire fructifier le plus possible» (id.).

En effet, la perfection à laquelle aspire Gérard s'acquiert d'abord dans l'accomplissement méticuleux - voire obsessionnel - du devoir d'état. A plusieurs reprises, le héros trace dans son journal de multiples horaires destinés à occuper saintement ses journées. L'observance rigoureuse de cette "loi du Père» devrait, pense-t-il, le conduire à la sainteté. En plus, les traditionnelles pénitences masochistes, toutes orchestrées d'après le refrain suivant: «je ferai tout mon possible pour plaire à Jésus en me mortifiant " (p. 11), font très tôt leur apparition et iront en s'accroissant.

Car la spiritualité est vécue de façon masochiste et terrifiante, bien dans le ton de l'époque. Un confrère meurt-il que Gérard note:

Il y a deux jours, il jouait avec ses camarades, jouissant d'une excellente santé et aujourd'hui, il est déjà passé au tribunal de Dieu! C'est un avertissement du ciel. Dieu a choisi celui-ci, pourquoi pas un autre, pourquoi pas moi. Donc, il faut toujours être prêt» (p. 12).

En fait, il ne semble y avoir qu'un moyen de contrer le courroux d'un Dieu aussi terrifiant: multiplier les mortifications: «oui, je veux anéantir mon corps, ne lui laisser que ce qu'il faut pour supporter mon âme» (p. 39). Tout manquement à cette "loi du Père», symbolisée par les «tables de mortification » établies par Gérard, entraîne un châtiment. II "faut suivre à la lettre mon règlement» (p. 28), ce leitmotiv revient constamment. De là à sauter à la mortification corporelle, il n'y a qu'un pas, que Gérard franchit facilement: 
« un cordon de nœuds de cuir serré autour des reins et sur les épaules, ça serre un peu, mais il n'y a pas encore de sang. Pourtant, il m'en faut verser du sang. il faudrait que je le donne tout entier, et encore cela ne suffirait pas» (p. 30).

Le but avoué de ces punitions est, en apparence, parfaitement justifié: «ainsi entraîné par la mortification, il sera facile de devenir apôtre " (id). Mais, en prime, l'exercice de ces sévices corporels promet pourtant d'indicibles félicités: «à chaque manquement que je ferai, je me punirai d'un coup de griffe quelque part sur mon corps. Je veux livrer une véritable bataille à moi-même. Pas de pitié. Cognons dur. La récompense sera belle» (p. 30).

Désormais, le journal sera le récit de cette lutte du héros contre ses pulsions mauvaises - personnifiées par le Diable qui le pousse à l'orgueil et à l'impureté ${ }^{22}$ - qu'il tente littéralement d'extirper de sa chair afin de plaire à Dieu. Dans cette croisade, le héros est aidé par l'exemple de modèles - le frère Lavallée, Saint Alphonse de Liguori et, surtout, Sainte Thérèse de l'Enfant Jésus - auxquels il tente de se conformer. S'il emprunte l'aspect «code civil» de sa spiritualité au frère Lavallée, l'amour hystérique de la carmélite exerce sur lui une profonde fascination. Comme elle, il rêve de s'offrir "en victime d'holocauste à l'amour miséricordieux» (p. 97).

Sa dévotion au Christ augmente de plus en plus et se traduit par des propos assez équivoques où se mêlent masochisme et passion:

Jésus, je veux vous aimer toujours. Je renouvelle l'offrande que je vous ai déjà faite. Comme Thérèse, je m'offre à vous comme victime d'amour. Je veux vous aimer jusqu'au martyre. Martyre caché d'aujourd'hui, de chaque jour; martyre de demain, sous le ciel orageux de la Chine. Je ne veux plus perdre de vue cet idéal; le martyre porte du ciel. Toute ma vie, me préparer pour en être digne. Me faire une âme pure, aimante, folle d'amour, comme celle de Thérèse (p. 119). ${ }^{23}$

Et à l'image de cette dernière, Gérard se met à cracher le sang le 2 janvier 1932, récompense suprême: «Donner mon sang en pleine vigueur de jeunesse, cela vaut bien le martyre lointain et problématique d'un vieillard de demain [...]. D'avance, Jésus, j'accepte tout, tout... et j'unis tout avec vos souffrances" (pp. 166-167). Ce sont les dernières lignes écrites par Gérard Raymond jusqu'à sa mort, six mois plus tard.

Si on scrute d'un point de vue psychanalytique ce lacis d'associations obsédantes, on peut dégager ce que Mauron nomme le «mythe personnel „24, c'est-à-dire la structure fantasmatique propre à chaque auteur. Chez Gérard Raymond, cette association de la souffrance à la passion amoureuse pour un objet de désir de sexe masculin, doublée d'une soumission (qui a toutes les allures d'une castration) à une imago paternelle sévère (le père et Dieu-le-Père) suggère un fantasme homosexuel. En effet, le moi (Gérard) se castre en niant son corps (ses pulsions) pour ne pas susciter la colère divine et, du même coup, conquérir Jésus ${ }^{25}$. D'ailleurs, les nombreux idéals du moi ${ }^{26}$ qui jalonnent le texte sont tous des individus qui ont renoncé à l'exercice de leur virilité - en entrant dans les ordres comme le frère Lavallée ou l'abbé Joseph Girard auteur de Vingt-deux années de martyre, livre de chevet de Gérard dans les derniers 
mois de sa vie - ou une femme qui se consume littéralement d'amour pour le Christ: Sainte Thérèse. Parallèlement, tous ces idéals du moi sont habités par des pulsions de mort assez évidentes: sans exception, ils appellent la mort de leurs prières et ne trouveront la paix que dans l'anéantissement total, qui est bien le masochisme poussé à l'extrême, le désir sexuel étant lié au désir de la mort ${ }^{27}$.

II est à peu près dans le cours normal des choses que ce genre de construction fantasmatique prenne racine dans une société fermée à l'autre sexe, comme les séminaires et les prisons. En revanche, ce qui paraît inquiétant, c'est que l'Église, par le biais de ses appareils idéologiques, ait favorisé ce type de construction fantasmatique jusqu'à l'ériger en «modèle» pour la jeunesse. Pouvons-nous supposer qu'une jeunesse «castrée» eut été plus facile à manœuvrer pour en faire des porte-paroles de l'idéologie de conservation? Car n'oublions pas que cette jeunesse qui militait dans les mouvements d'action catholique se livrait petit à petit à une remise en question du pouvoir clérical. De plus, au point de vue politique, il y avait des remous dans la société québécoise, provoquées par la fondation de l'Action libérale nationale en 1934. Celle-ci devient le catalyseur des opposants au régime Taschereau, appuyé par le clergé, en prônant en particulier la libération économique et sociale des Québécois. La suprématie sociale de l'Église s'effrite de plus en plus et ne suffit pas à assumer la survie de la nation ${ }^{28}$.

On peut aussi s'interroger sur la coïncidence entre le fantasme de Gérard et le désir d'une fraction de la société. Si on en juge par les témoignages publiés en appendice, il semble que le volume ait eu du succès auprès des membres de communautés religieuses et de la fraction la moins progressiste de la classe ouvrière et étudiante. Il s'agit manifestement d'un groupe qui voit lentement se désagréger sa puissance (le clergé) et qui appréhende le bouleversement de ses conditions de vie (la portion conservatrice des classes étudiante et ouvrière). Mais il est bien significatif que cette structure fantasmatique ne semble pas rejoindre ceux à qui elle était destinée d'emblée: la jeunesse des mouvements d'Action catholique. Ceci témoigne bien de la faille au sein de la puissance cléricale - qui ne parvient plus à imposer son modèle homosexuel masochiste et, disons le, fasciste ${ }^{29}$ - même s'il faudra une autre génération pour la jeter dans l'ombre.

Ce travail, dont le but premier était d'esquisser la filiation entre une structure fantasmatique et ses racines sociales, a négligé plusieurs aspects. L'analyse détaillée du Journal de Gérard Raymond ferait à elle seule l'objet d'un livre, même si cette œuvre ne semble pas justifier qu'on y consacre autant d'énergie. En revanche, il serait intéressant d'effectuer l'inventaire et l'analyse de notre "Bibliothèque Bleue» québécoise. En s'aidant de la psychanalyse et de la sociologie, peut-être pourra-t-on mieux comprendre pourquoi ce que Mario Fontaine nomme ala bondieuserie ${ }^{30}$ a exercé une si profonde influence sur la société québécoise. 
1. Robert Blondin, Chers nous autres. Un siècle de correspondance québécoise, Montréal, éd. VLB, 1978, 2 vol.

2. Pierre Crépeau et al., L'album de famille des Québécois 1870-1970, Montréal, éditions Québécoises, 1972.

3. Pierre Pagé et Renée Legris, Le comique et l'humour à la radio québécoise. Montréal, éd. La Presse, 1976.

4. Line Ross et Hélène Tardif, Le téléroman québécois 1960-1971. Université Laval, Laboratoire de recherches sociologiques, 1975.

5. Chantal Hébert, Le théâtre burlesque au Québec; étude d'un divertissement populaire, Montréal, Leméac, 1980 (à paraître).

6. Mario Fontaine, Tout sur les p'tits journaux z'artistiques, Montréal, éd. Quinze. 1978.

7. Ixe-13 (Je me souviens des Aventures etranges de l'agent /xe-13, I'as des espions canadiens), Montréal, Office National du Film, 1972.

8. Voir à ce sujet: Ixe-13, un cas type de roman de masse au Québec, numéro de la revue Études littéraires, Québec, Presses de l'Université Laval, vol. 12, $\mathrm{n}^{\circ} 2$, août 1979, $301 \mathrm{p}$. Un collectif intitulé Le ohénomène /xe-13 paraîtra dans la collection *Vie des Lettres québécoises» aux Presses de l'Université Laval en 1981. Le groupe effectue présentement une recherche sur la littérature populaire en fascicules: 1940-1970.

9. Jean Simard, Jocelyne Milot et René Bouchard, Un patrimoine méprisé; la religion populaire des Québécois, Montréal, Hurtubise HMH, 1979.

10. Victor-Lévy Beaulieu, Manuel de la petite littérature du Québec, Montréal, éd. de l'Aurore, 1974.

11. «Répertoire littéraire de soumission et de statisme qu'on peut dépouiller dans un ordre qui confond à dessein la mission des clercs et des laïcs pour les dresser à l'amour sublimé d'une puissance autochtone forte et à l'homogénéisation sociale conforme à l'idéologie dominante». Brigitte Sicard, «L'enjeu d'un concept: le nationalisme littéraire des années $30 \%$, Voix et lmages, vol. III, $n^{\circ} 1$, septembre 1977, p. 80.

12. Ce qu'affirme Geneviève Bollême à propos des thèmes de la bibliothèque bleue s'applique aussi à notre paralittéraire religieuse: «c'est le désir de vivre mieux qui. par delà les thèmes, est le plus fort en ce qu'il assure la cohérence, l'homogénéité de la bibliothèque bleue; et en fait aussi l'originalité. II faut lire les titres pour ce qu'ils content et non pas seulement comme un répertoire. Ils sont langs, détaillés, présentent un résumé, contiennent parfois une sorte de moralité. Ceci dans les premiers livrets et les livrets romanesques principalement. C'est-à-dire qu'en général, ils annoncent qu'une conduite noble, vaillante, généreuse est récompensée par le gain d'un royaume, d'une vie heureuse». Geneviève Bollême, $\mathrm{La}$ Bible blecue; anthologie d'une littérature «populaire», Paris, Flammarion, 1975, p. 36 .

13. Des cuvres marginales comme Thérèse Gélinas du Père Eugène Nadeau, publiée chez Beauchemin en 1936, et Jean Olscamp de l'abbé Léopold Taillon, publiée à I'Atelier en 1957 ont connu respectivement un tirage de 35000 et 23000 exemplaires ainsi que trois éditions successives. Certaines œuvres ont même été traduites: la biographie écrite par le Révérend O.A. Boyer, Couronnée d'épines Marie-Rose Ferron, surnommée la Petite Rose, la stigmatisée de Woonsocket parue à Montréal en 1947 a été traduite en anglais et publiée sous le titre She wears a Crown of Thorns par Benziger Brother, New York, en 1949. Celle composée par le Père Antonio Dragon, Toujours plus haut, publiée par la Maison Saint-Joseph en 1925, a été traduite en anglais sous le titre Excelsior et publiée par Loyola University Press en 1930. Nous ne possédons pas encore de renseignements précis sur des œuvres à succès comme le Journal de Gérard Raymond et le Cantique d'action de gráce ou chant d'amour de Mère Marie-Sainte-Cécile-de-Rome. Mentionnons pourtant que cette dernière ceuvre a connu au moins deux éditions canadiennes et deux éditions françaises. Or, d'après une enquête effectuée en 1962, «les romans sont le plus souvent tirés à 3000 ou 3500 exemplaires et les 
éditeurs considèrent comme un succès relatif une vente atteignant $2000 \mathrm{exem}$. plaires [...]. Les gros tirages, ceux qui permettraient de combler un déficit, sont exceptionnels. II est significatif, de ce point de vue, de noter qu'on considère, chez la plupart de nos informateurs, qu'un roman devient un best-seller s'il atteint les 5000 exemplaires». Nous soulignons. Claude Corriveau et al., "Une enquête: le statut de l'écrivain et la diffusion de la littérature", Littérature et société canadiennes-françaises, Québec, Presses de I'Université Laval, 1964, p. 93.

14. op. cit.

15. Corine Rocheleau, Hors de sa prison; extraordinaire histoire de Ludivine Lachance, l'infirme des infirmes, sourde, muette et aveugle, Montréal, Arbour et Dupont, 1927, $270 \mathrm{p}$.

16. Encore faudrait-il nuancer cette affirmation. Le Journal de Gérard Raymond, préfacé par le Cardinal Villeneuve, est expressément dédié a ala jeunesse des écoles, collèges, séminaires et universités qui se groupe dans les mouvements spécialisés d'Action catholique» (p. 6). Or, on sait que la J.E.C., pur produit des années 30, marque dans la société québécoise un premier bris dans le vaste réseau clérical: «on doit retenir davantage ce qu'a été ce mouvement comme nouveau cadre de référence pour une foule de valeurs qui auparavant étaient inexistantes dans le milieu ou qui étaient tout simplement censurées par des représentations idéologiques plus restrictives». André-J. Bélanger, Rupture et constantes: Quatre idéologies du Québec en éclatement, Montréal, Hurtubise $\mathrm{HMH}, 1977$, p. 37. II y aurait sûrement une étude à faire sur les rapports entre le clergé et la J.E.C., le premier tentant de faire du second un de ses appareils idéologiques et complètement aveuglé (du moins la dédicace du Journal le laisse croire) sur le fait que l'Action catholique - dont la J.E.C. est une composante - se sert de l'Église comme point d'appui pour un mouvement d'émancipation qui ira en s'élargissant au cours des années 40 et 50.

17. C'est d'ailleurs ce que tente en partie Philippe Lejeune dans ses travaux sur l'autobiographie où il démontre que la canonisation de l'autobiographie ne va pas sans rapport avec un autre mythe canonique, psychanalytique celui-là. Cf. Le pacte autobiographique, Paris, Seuil, 1975.

18. On ne peut s'empêcher $d^{\prime} y$ voir des «ancêtres» des romans d'amour en fascicules publiés et diffusés pendant les années quarante et cinquante, qui semblent privilégier une structure fantasmatique voisine (soumission à l'autorité et masochisme). Voir à ce sujet: Caroline Barrett, «/xe-13, un roman sentimental "Études littéraires, vol. $12, n^{\circ} 2$, août 1979 , pp. 235-245.

19. Vraisemblablement «nos» collégiens, «nos» séminaristes et, peut-être, «nos» universitaires, ardents partisans de la révolution tranquille qui sonnera le glas de la puissance cléricale. Car ce désir, bien mal endigué par le clergé, finira pourtant par le submerger et provoquer l'affranchissement des tutelles traditionnelles que les clercs, de leur propre aveu, redoutent tellement. Cf. les nombreux éditoriaux de Mgr Paquet dans L'Action catholique et les pamphlets de l'École sociale populaire.

20. Afin de distinguer chez Gérard Raymond l'auteur du narrateur, nous désignerons par \& Gérard Raymond * l'auteur et par «Gérard " le narrateur qui par l'acte d'écrire se projette dans le texte.

21. II s'agit d'un obscur saint québécois (1899-1922) dont la biographie a été rédigée par nul autre que le Cardinal Villeneuve. Le frère Lavallée est qualifié par Gérard Raymond de «modèle de la jeunesse édudiante» (p. 12). II lui emprunte un certain nombre de pratiques obsessionnelles plus ou moins masochistes: « maintenant, je veux l'imiter, me tracer un plan de bataille analogue au sien, qui a si bien réussi [Le frère Lavallée est mort noyé!] Je veux lui emprunter sa méthode pour obtenir le recueillement, brider l'imagination, réduire l'amour-propre, pratiquer la mortification des sens. Je veux me faire un tableau de ses pratiques de dévotion (P. 52).

22. «Quelques instants de répit, puis le diable revient à la charge. Il en a de la volonté et de la persévérance, luil Cette fois, c'est pour $\mathrm{m}$ 'enorgueillir de la ferveur que je peux avoir dans mes prières» (p. 23). «Ce matin, je me suis levé et habillé en vrai chrétien. Pendant ce temps, le diable dans son coin a essayé de me faire des petites 'suggestions' $"$ (p. 22). 
23. II est bien intéressant qu'au plus fort de son délire, Gérard s'identifie à ce point à une femme (Thérèse) et avoue clairement son désir de séduction homosexuelle. II faut aussi préciser que Gérard Raymond conservait sur lui une image du Christ où il avait inscrit ces mots: «Toi, du moins, aime-moi".

24. Voir à ce sujet: Charles Mauron, Des métaphores obsédantes au mythe personnel, Paris, José Corti, 1971, pp. 32-33.

25. Dans le jargon psychanalytique, l'idéal du moi est un modèle auquel le sujet cherche à se conformer, consciemment ou non. Dans le cas de Gérard, c'est toutà-fait conscient. Cf. Freud, «Pour introduire le narcissisme», La vie sexuelle, Paris, Presses Universitaires de France, 1972, pp. 81-106 où il démontre I'origine narcissique de l'idéal du moi.

26. Et le refoulement de l'agressivité pratiqué par le héros ne peut qu'entraîner un retournement sur soi de cette agressivité: le masochisme. De fait, la soumission de Gérard à son a règlement » le conduit tout droit au sanatorium. II n'est pas indifférent qu'il soit mort justement de tuberculose. André Crouzatier a tenté, à partir de psychanalyses de tuberculeux, d'isoler un certain nombre de facteurs déclenchants : frustrations, absence affective du père, mère étouffante et, surtout, incompatibilité entre le monde fantasmatique du sujet et la réalité externe. Les recherches de Crouzatier adessinent l'image d'une personnalité proche de la névrose de caractère du type narcissique dans laquelle les fixations apparaissent plus déterminantes que les régressions, ce qui confère à ces malades rebelles une composante d'arriération affective. Tout se passe comme si ces sujets étaient incapables de développer leur antinarcissisme». «Psychanalyse et psychothérapie des tuberculeux», Revue française de psychanalyse, mai-juin 1969, p. 503.

27. Voir à ce sujet l'article «pulsion de mort» dans Jean Laplanche et J.-B. Pontalis, Vocabulaire de la psychanalyse, Paris, Presses Universitaires de France, 1968. pp. 371-377.

28. Cf. Everett C. Hughes, Rencontre de deux mondes: la crise d'industrialisation du Canada français, Montréal, Boréal Express, 1972 et Denis Monière, Le développement des idélogies au Québec. Montréal, éditions Québec-Amérique, 1977. pp. 263-290.

29. Ce lien entre un tel mysticisme à composante masochiste et le fascisme a été souligné par Maria-Antonietta Macciocchi: «c'est dans le délire de nombreuses religieuses qui se croient les fiancées du Christ et qui choisissent d'autres voies sexuelles telles que le martyre masochiste que l'on peut joindre ces deux racines, celles du mysticisme et celle de la pulsion fasciste». "Sexualité féminine dans l'idéologie fasciste», Sexualité et politique, Paris, Union Générale d'Édition, coll. 10/18, 1975, p. 257.

30. Mario Fontaine, op. cit., p. 159. Et la survivance de cette «bondieuserie» dans un chef politique tel que Claude Ryan qui, fort de son passé de militant d'Action catholique et d'émissaire de la «main de Dieu», jouit d'un très grand crédit auprès d'une fraction importante de la société québécoise. 